\title{
Blast Injury
}

National Cancer Institute

\section{Source}

National Cancer Institute. Blast Injury. NCI Thesaurus. Code C34427.

The result of physical trauma sustained in an explosion. 